\title{
A Fully 3-D BIE Evaluation of the Resistance and Inductance of On-Board and On-Chip Interconnects
}

\author{
Martijn Huynen, Daniël De Zutter, and Dries Vande Ginste \\ Electromagnetics Group/IDLab, Department of Information Technology, Ghent University/imec \\ Gent, Belgium \\ martijn.huynen@ugent.be
}

\begin{abstract}
In this contribution, the resistance and inductance of 3-D interconnects are obtained through a full-wave approach. By solving a free space boundary integral equation (BIE) combined with a fully 3-D differential surface admittance operator in a circuit framework, an effective procedure to study finite conductivity interconnects is presented. The accuracy of the proposed method in characterizing 3-D interconnects is demonstrated through an on-board and an on-chip example.
\end{abstract}

Index Terms-interconnect modeling, boundary integral equation (BIE), 3-D surface admittance.

\section{INTRODUCTION}

The continuing search for smaller and faster electronic circuits to satisfy the needs of our information-driven global society has led to increasingly complex structures in both printed circuit board (PCB) and integrated circuit (IC) technologies. One example of such an innovation is the rise of three-dimensional (3-D ICs) [1]. The increased density of components and interconnects poses many challenges, not in the least in terms of signal integrity. Proximity of various signal lines and noisy circuits to interconnects introduces numerous detrimental phenomena such as crosstalk and signal distortion. These effects only get amplified by the cluttered electromagnetic environment and the high frequency spectral content of digital signals caused by increasing data rates. Accurate modeling of circuits and especially interconnects becomes ever more indispensable in modern design tools.

Over the years, various models and techniques to model interconnects have been proposed and developed. 3-D approaches can generally be categorized as either volume or surface discretization methods. A example from the first category is the finite element method (FEM) [2]. The main drawback of this method for interconnect modeling is the fact that the volume mesh size has to be of the order of the skin depth and thus leads to an enormous number of unknowns.

Boundary integral equation (BIE) based methods such as the method of moments (MoM) are inherently less susceptible to this problem as only the surface is meshed. However, tackling the interior problem for high conductive media poses challenges of its own such as the calculation of the interaction integrals [3] or guaranteeing the accuracy of the iterative solution [4]. Therefore, another popular approach is to replace the inner material and invoke an additional relation between the fields on the surface to adequately capture the influence of the replaced material. A widely used choice is the Leontovich boundary condition which imposes a local relation between the surface current density and the electric field. Various approaches to expand this condition to a more global relation make use of volume integral equations [5] or boundary integral equations [6] in the cross-section.

In [7], for the first time in literature, a 2-D differential surface admittance operator was presented to effectively model the skin effect. This operator was leveraged in [8] to compute the per unit of length (p.u.1.) parameters of general 2-D multiconductor transmission lines. Further, this method has been successfully applied to various interconnect problems [9], [10]. The 2-D operator of [7] was also exploited in [11] for the modeling of 3-D interconnects in an approximate way. This was done by treating each separate 2-D segment of the 3-D interconnect by means of the 2-D operator and calculating the interaction between the segments with a BIE.

In contrast, in this paper, we propose a fully 3-D differential surface admittance operator. In order to model 3-D interconnects, this operator is constructed for cuboids instead of cylinders as reported in [12]. Plugging this operator into the electric field integral equation and combining it with a circuit interpretation permits the characterization of the resistance and inductance of PCB and IC interconnects.

\section{Formulation OF THE METHOD}

Consider the geometry depicted in Fig. 1(a). Volume $\mathcal{V}$ with arbitrary dimensions and boundary surface $\mathcal{S}$ consist of a nonmagnetic, homogeneous medium characterized by a wavenumber $k$. This region is enclosed by the background medium $\mathcal{V}_{0}$ characterized by its own wavenumber $k_{0}$ (presuming once again a non-magnetic material). The time-harmonic fields ( $e^{j \omega t}$ dependence) impinging on $\mathcal{V}$ from sources located in $\mathcal{V}_{0}$ are given by $\left(\mathbf{e}_{i}, \mathbf{h}_{i}\right)$ and result in the total field distribution $\left(\mathbf{e}_{0}, \mathbf{h}_{0}\right)$ in the background medium. Inside $\mathcal{V}$, the sources induce the fields $\left(\mathbf{e}_{1}, \mathbf{h}_{1}\right)$.

The situation in Fig. 1(b) depicts the same geometry but the material inside $\mathcal{V}$ is replaced by that of the background medium $\mathcal{V}_{0}$. A surface current density $\mathbf{j}_{s}$ is introduced on the boundary $\mathcal{S}$ to preserve the total fields $\left(\mathbf{e}_{0}, \mathbf{h}_{0}\right)$ outside $\mathcal{V}$ while the field distribution inside this volume will change to $(\mathbf{e}, \mathbf{h})$.

In order to solve for the field distributions in the equivalent problem, we express the scattered electric field by means of the magnetic vector potential a and electric scalar potential $\phi$ :

$$
\mathbf{e}_{0}-\mathbf{e}_{i}=-\nabla \phi-j \omega \mathbf{a} .
$$




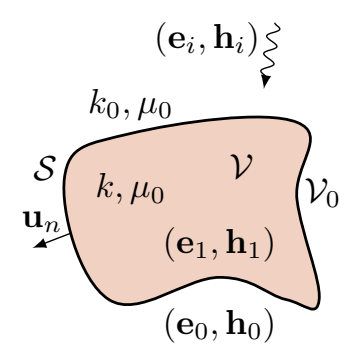

(a) Original situation.

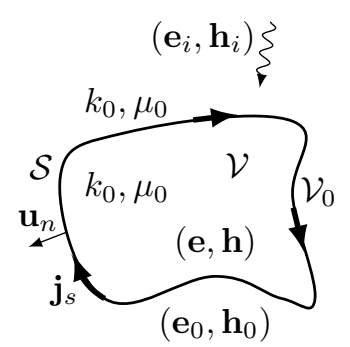

(b) Equivalent situation.
Fig. 1. Problem statement. (a) A homogeneous volume $\mathcal{V}$ with boundary $\mathcal{S}$ is surrounded by another homogeneous volume $\mathcal{V}_{0}$. In (b), the inner material is replaced by the background medium and a surface current density $\mathbf{j}_{s}$ is introduced on $\mathcal{S}$. All materials are presumed to be non-magnetic.

Generally, this equation can be solved by discretizing $\mathcal{S}$ by means of a triangular and/or rectangular mesh and employing basis and test functions to transform (1) into a matrix equation. As all our examples deal with cuboid meshes, we will utilize rooftop functions (see Fig. 2). Given the common edge $e_{i}$ between two rectangles, a rooftop is defined as

$$
\mathbf{b}_{i}(\mathbf{r})= \begin{cases}{\left[\left(\mathbf{r}-\mathbf{r}^{+}\right) \cdot \mathbf{u}^{+}\right] \mathbf{u}^{+} / A_{i}^{+},} & \text {if } \mathbf{r} \in \mathrm{R}_{i}^{+} \\ {\left[\left(\mathbf{r}^{-}-\mathbf{r}\right) \cdot \mathbf{u}^{-}\right] \mathbf{u}^{-} / A_{i}^{-},} & \text {if } \mathbf{r} \in \mathrm{R}_{i}^{-} \\ 0, & \text { elsewhere }\end{cases}
$$

with $\mathrm{R}_{i}^{ \pm}$both support rectangles, $A_{i}^{ \pm}$their respective area, $\mathbf{r}^{ \pm}$ corners not belonging to $e_{i}$ and $\mathbf{u}^{ \pm}$the direction of the rooftop on the relevant rectangle.

We now test (1) with these functions $\mathbf{b}_{i}$. If we also expand the unknown electric field $\mathbf{e}_{0}$ into the same basis functions:

$$
\mathbf{e}_{0}=\sum_{j} E_{0, j} \mathbf{b}_{j}
$$

the first term becomes $\overline{\bar{G}} \mathbf{E}_{0}$ where the vector $\mathbf{E}_{0}$ contains all expansion coefficients of $\mathbf{e}_{0}$ and $\overline{\bar{G}}$ is the relevant Gram matrix:

$$
\overline{\bar{G}}_{i, j}=\int_{\mathcal{S}_{i}} \mathbf{b}_{i} \cdot \mathbf{b}_{j} \mathrm{~d} S .
$$

The second term in the equation simply turns into a vector $\mathbf{E}_{i}$ containing elements which represent the impinging electric field weighted with $\mathbf{b}_{i}$. The first term in the right-hand side of (1) can be written as

$$
\begin{aligned}
\int_{\mathcal{S}_{i}} \nabla \phi \cdot \mathbf{b}_{i} \mathrm{~d} S & =\int_{\mathcal{S}_{i}} \nabla\left(\phi \mathbf{b}_{i}\right) \mathrm{d} S-\int_{\mathcal{S}_{i}} \phi \nabla \cdot \mathbf{b}_{i} \mathrm{~d} S \\
& =\oint_{c_{i}} \phi \underbrace{\mathbf{u}_{n} \cdot \mathbf{b}_{i}}_{=0} \mathrm{~d} c-\left[\int_{R_{i}^{+}} \frac{\phi}{A_{i}^{+}} \mathrm{d} S-\int_{R_{i}^{-}} \frac{\phi}{A_{i}^{-}} \mathrm{d} S\right] \\
& =V_{i}^{-}-V_{i}^{+},
\end{aligned}
$$

with $V_{i}^{ \pm}$the average potential over the two rectangles $R_{i}^{ \pm}$ supporting $\mathbf{b}_{i}$. In order to reduce the first surface integral on the right-hand side of (5) to a line integral over the closed boundary $c_{i}$ of $\mathcal{S}_{i}$ with outward pointing normal $\mathbf{u}_{n}$, we have employed the divergence theorem.

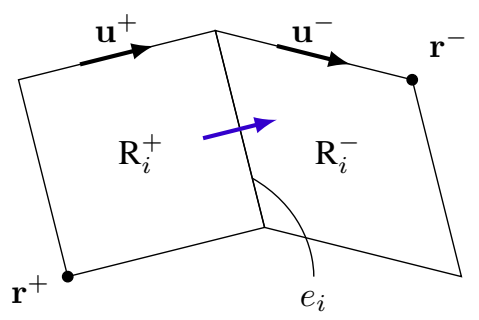

Fig. 2. Rooftop basis function.

The last term on the right-hand side can also be discretized into a matrix equation by expanding the surface current density into rooftop functions and introducing $G\left(\mathbf{r}, \mathbf{r}^{\prime}\right)$, the Green's function of the equivalent problem, to obtain:

$$
\int_{\mathcal{S}_{i}} \mathbf{a} \cdot \mathbf{b}_{i} \mathrm{~d} S=\sum_{j} I_{j} \mu_{0} \int_{\mathcal{S}_{i}} \int_{\mathcal{S}_{j}} G\left(\mathbf{r}, \mathbf{r}^{\prime}\right) \mathbf{b}_{i} \cdot \mathbf{b}_{j} \mathrm{~d} S \mathrm{~d} S^{\prime}=\overline{\bar{L}} \mathbf{I}
$$

where the vector $\mathbf{I}$ collects all the expansion coefficients of $\mathbf{j}_{s}$.

The discretized equivalent of (1) is thus

$$
\overline{\bar{G}} \mathbf{E}_{0}-\mathbf{E}_{i}=-j \omega \overline{\bar{L}} \mathbf{I}+\mathbf{V}^{+}-\mathbf{V}^{-} .
$$

We can now eliminate one set of unknowns by introducing the fully 3-D differential surface admittance operator that links $\mathbf{j}_{s}$ and $\mathbf{e}_{0}$, i.e., $\mathbf{j}_{s}=\mathcal{Y} \mathbf{e}_{0}$ [12]. This operator is given by the following expression (with the contrast parameter $\left.\eta=\left(k^{2}-k_{0}^{2}\right) / j \omega \mu_{0}\right)$ :

$$
\mathbf{j}_{s}=\eta \sum_{l}\left[\frac{\left|k_{l}\right|^{2} \int_{\mathcal{S}}\left(\mathbf{u}_{n} \times \mathbf{e}_{0}\right) \cdot \mathbf{h}_{l}^{*} \mathrm{~d} S}{\left(k_{l}^{2}-k^{2}\right)\left(k_{l}^{2}-k_{0}^{2}\right) \mathcal{N}_{l}^{2}}\right]\left(\mathbf{u}_{n} \times \mathbf{h}_{l}\right),
$$

with $\mathbf{h}_{l}$ the magnetic eigenmodes of $\mathcal{V}$ with a perfect electric conductor (PEC) surface $\mathcal{S}$ with wavenumber $k_{l}$ and normalization constant $\mathcal{N}_{l}$. For a cuboid with dimensions $\{a, b, c\}$, the TE eigenfunctions and their corresponding wavenumbers are defined as

$$
\begin{aligned}
\mathbf{h}_{l m n}^{\mathrm{TE}} & =\nu\left[\lambda \sin (\lambda x) \cos (\mu y) \mathbf{u}_{x}+\mu \cos (\lambda x) \sin (\mu y) \mathbf{u}_{y}\right] \\
& \cdot \cos (\nu z)-\left(\lambda^{2}+\mu^{2}\right) \cos (\lambda x) \cos (\mu y) \sin (\nu z) \mathbf{u}_{z}, \\
k_{l m n}^{2} & =\left(\frac{l \pi}{a}\right)^{2}+\left(\frac{m \pi}{b}\right)^{2}+\left(\frac{n \pi}{c}\right)^{2}=\lambda^{2}+\mu^{2}+\nu^{2},
\end{aligned}
$$

while the TM modes (with the same wavenumber) are

$$
\begin{aligned}
\mathbf{h}_{l m n}^{\mathrm{TM}} & =k_{l m n}\left[\mu \sin (\lambda x) \cos (\mu y) \mathbf{u}_{x}-\lambda \cos (\lambda x) \sin (\mu y) \mathbf{u}_{y}\right] \\
& \cdot \cos (\nu z) .
\end{aligned}
$$

Plugging the discretized version of the differential surface admittance operator, i.e., $\overline{\bar{G}} \mathbf{I}=\overline{\bar{Y}} \mathbf{E}_{0}$ into (7), one finds

$$
\left(\overline{\bar{G}} \overline{\bar{Y}}^{-1} \overline{\bar{G}}\right) \mathbf{I}-\mathbf{E}_{i}=-j \omega \overline{\bar{L}} \mathbf{I}+\mathbf{V}^{+}-\mathbf{V}^{-}
$$

This final equation can be solved in various ways but in our technique we interpret the equation as a circuit (shown in Fig. 3 for two edges). This approach enables the use of circuit solvers to evaluate (12) and facilitates the addition of discrete circuit components. The results in this paper are calculated employing the circuit solver LTspice ${ }^{\circledR}$ [13]. 


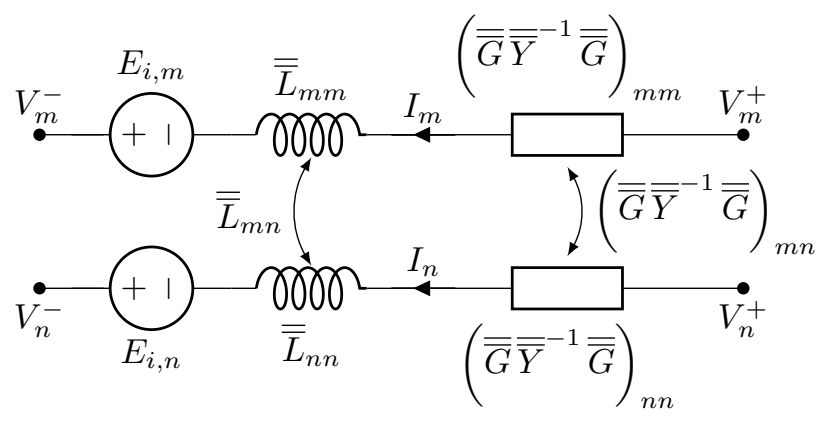

Fig. 3. Equivalent circuit interpretation of (12).

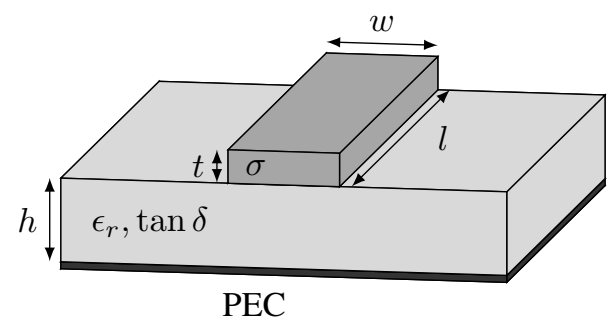

Fig. 4. Microstrip configuration on a RO4350B RF substrate $\left(\epsilon_{r}=\right.$ $3.48, \tan \delta=0.003, w=530 \mu \mathrm{m}, t=35 \mu \mathrm{m}, h=250 \mu \mathrm{m}, l=10 \mathrm{~mm}$, $\left.\sigma=5.8 \cdot 10^{7} \mathrm{~S} / \mathrm{m}\right)$.

\section{NUMERICAL EXAMPLES}

\section{A. PCB Validation}

Consider a PCB microstrip configuration as presented in Fig. 4. A copper conductor $\left(\sigma=5.8 \cdot 10^{7} \mathrm{~S} / \mathrm{m}\right)$ and a perfect electric ground plane are placed on either sides of a RO4350B substrate $\left(\epsilon_{r}=3.48, \tan \delta=0.003\right)$. The height of this substrate is $h=250 \mu \mathrm{m}$ while the dimensions of the strip are $530 \mu \mathrm{m} \times 35 \mu \mathrm{m} \times 10 \mathrm{~mm}$.

The resistance per unit of length (p.u.l.), simply obtained by dividing the total resistance of the strip by its finite length $l$, is shown in Fig. 5(a) together with a 2-D reference solution obtained through the technique described in [8]. The lowfrequency value converges to the Pouillet value while we clearly see the influence of the skin effect for increasing frequencies. Furthermore, we observe an excellent agreement between the $2 \mathrm{D}$ results and our proposed method.

In Fig. 5(b) the inductance per unit of length is plotted for both methods. For this characteristic we observe a much stronger influence of the finite length of the microstrip. The difference between the curves is strongest for the lowfrequency inductance where we observe a difference of $3 \%$ between both results. However, tests have shown that increasing the length of the microstrip raises the curve towards the 2-D result, as expected.

\section{B. IC Application}

The second example characterizes a rectangular, copper loop as found in ICs. The dimensions are displayed in Fig. 6 (all in $\mu \mathrm{m}$ ) with one length being variable and ranging from 10 to $30 \mu \mathrm{m}$. The relevant parameters of this structure are calculated across the $1 \mu \mathrm{m}$ gap. Firstly, the resistance and inductance our

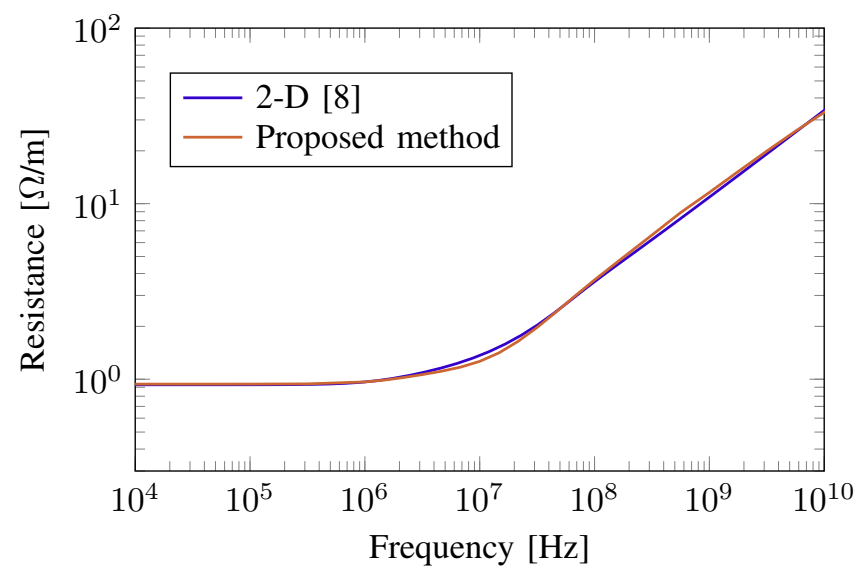

(a) P.u.l. resistance in $\Omega / \mathrm{m}$.

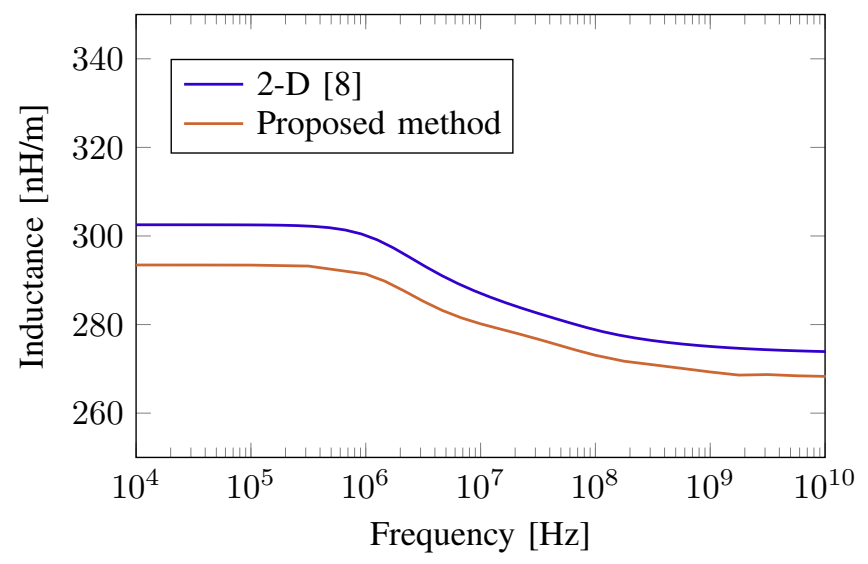

(b) P.u.1. inductance in $\mathrm{nH} / \mathrm{m}$.

Fig. 5. P.u.l. resistance and inductance of the microstrip shown in Fig. 4.

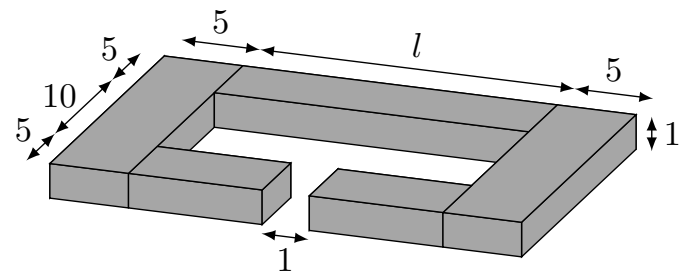

Fig. 6. Copper loop $\left(\sigma=5.8 \cdot 10^{7} \mathrm{~S} / \mathrm{m}\right)$ with one side of variable length $l$. All dimensions shown are given in $\mu \mathrm{m}$.

computed for one specific value of $l$, i.e., $l=20 \mu \mathrm{m}$, and compared to results from ANSYS HFSS (Fig. 7). For low frequencies, we observe excellent agreement of both resistance and inductance. For high frequencies, on the other hand, the results diverge. The unphysical kink in the inductive response of HFSS indicates the expected poor volume meshing of the good conductor; a problem mitigated by our BIE approach.

Finally, the resistance and inductance for various values of $l$ are plotted on both graphs of Fig. 8. Careful inspection of the low-frequency value of the resistances shows that the obtained values are smaller than the DC resistance of a single cuboid with the same total length as the loop (see the selected results in Tab. I). This can be attributed to an accurate 3-D 


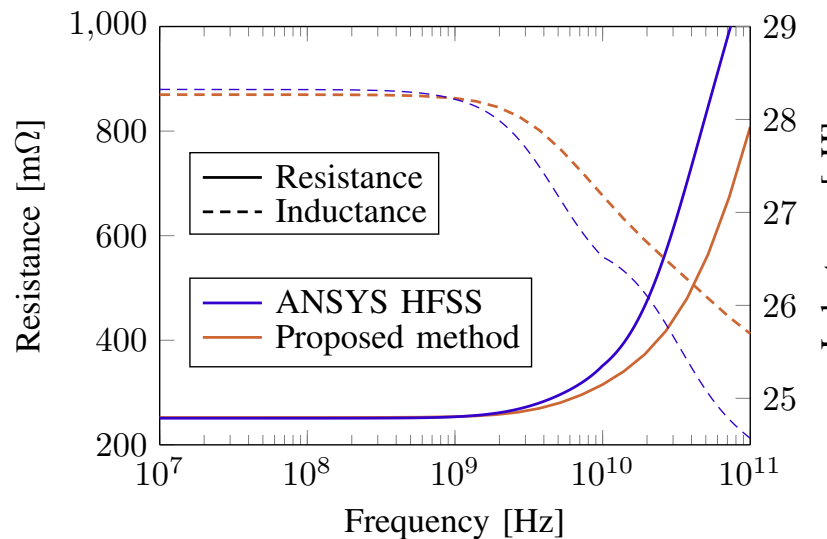

Fig. 7. Resistance and inductance of the loop in Fig. 6 for $l=20 \mu \mathrm{m}$ for the proposed method and ANSYS HFSS.

TABLE I

DC RESISTANCE OF A STRAIGHT COPPER CUBOID AND LOOP (SEE FIG. 6) FOR VARIOUS VALUES OF $l$.

\begin{tabular}{lcc}
\hline$l[\mu \mathrm{m}]$ & cuboid $[\mathrm{m} \Omega]$ & loop $[\mathrm{m} \Omega]$ \\
\hline 10 & 203.5 & 175.5 \\
15 & 237.9 & 210.2 \\
20 & 272.4 & 245.0 \\
25 & 306.9 & 279.8 \\
30 & 341.4 & 314.6 \\
\hline
\end{tabular}

modeling of the corners present in the loop. For the higher frequencies we note that the curves are more tightly packed. The smaller value of $l$ makes the loop more compact and brings the various conductors closer together. This strengthens the proximity effect and causes the smaller loop's resistance to start increasing at an earlier frequency than for the larger loop.

\section{CONCLUSIONS}

In this contribution, a fully 3-D differential surface admittance operator based on the eigenfunctions of cuboids was presented in a boundary integral equation framework to model 3-D interconnects. The resistance and inductance of examples from both PCB and IC applications were efficiently and accurately extracted through a circuit interpretation of the integral equation, clearly demonstrating the influence of pertinent phenomena such as the skin effect and the proximity effect.

\section{REFERENCES}

[1] E. Sicard, W. Jianfei, R. J. Shen, E. P. Li, E. X. Liu, J. Kim et al., "Recent advances in electromagnetic compatibility of 3D-ICs - Part I," IEEE Electromagn. Compat., vol. 4, no. 4, pp. 79-89, 2015.

[2] J. Jin, The Finite Element Method in Electromagnetics. Wiley, 2015.

[3] J. Peeters, I. Bogaert, and D. De Zutter, "Calculation of MoM interaction integrals in highly conductive media," IEEE Trans. Antennas Propag., vol. 60, no. 2, pp. 930-940, Feb 2012.

[4] M. Gossye, M. Huynen, D. Vande Ginste, D. De Zutter, and H. Rogier, "A Calderón preconditioner for high dielectric contrast media," IEEE Trans. Antennas Propag., vol. 66, no. 2, pp. 808-818, Feb 2018.

[5] M. Al-Qedra, J. Aronsson, and V. Okhmatovski, "A novel skin-effect based surface impedance formulation for broadband modeling of 3-D interconnects with electric field integral equation," IEEE Trans. Microw. Theory Techn., vol. 58, no. 12, pp. 3872-3881, Dec 2010.

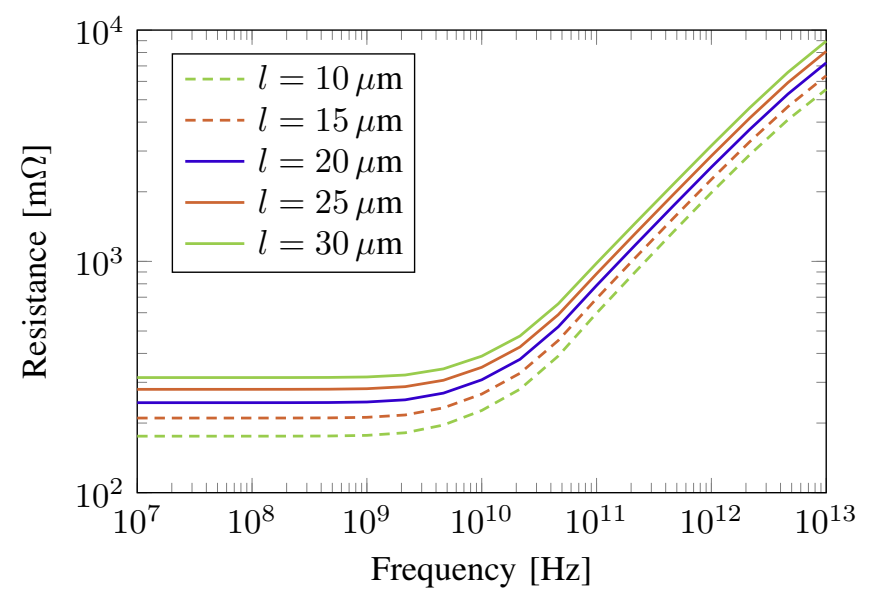

(a) Resistance in $\mathrm{m} \Omega$.

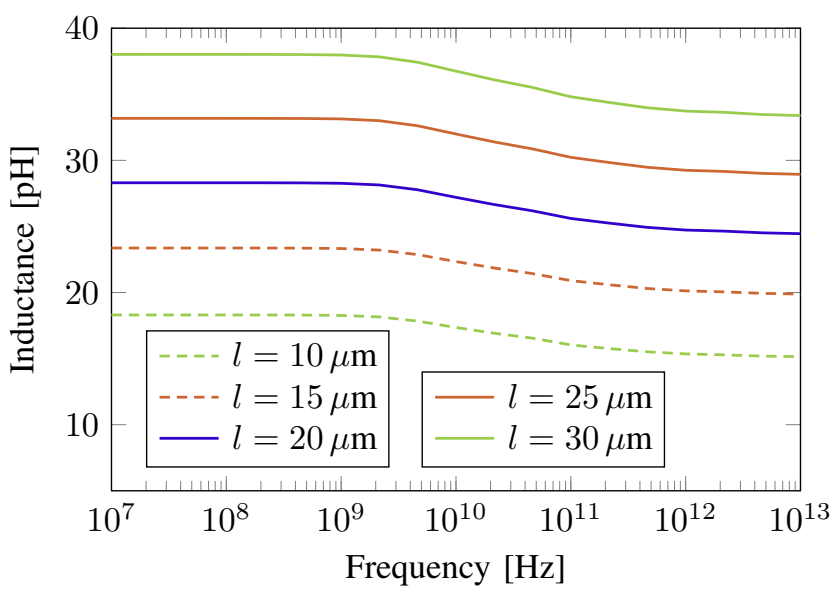

(b) Inductance in $\mathrm{pH}$.

Fig. 8. Resistance and inductance of the loop shown in Fig. 6 for various values of the parameter $l$.

[6] Y. Zhao, F. Ling, and J. Mao, "Novel surface impedance modeling for broadband parameter extraction of 3-D interconnects," IEEE Microw. Wireless Compon. Lett., vol. 27, no. 1, pp. 7-9, Jan 2017.

[7] D. De Zutter and L. Knockaert, "Skin effect modeling based on a differential surface admittance operator," IEEE Trans. Microw. Theory Techn., vol. 53, no. 8, pp. 2526-2538, Aug 2005.

[8] T. Demeester and D. De Zutter, "Quasi-TM transmission line parameters of coupled lossy lines based on the Dirichlet to Neumann boundary operator," IEEE Trans. Microw. Theory Techn., vol. 56, no. 7, pp. 16491660, July 2008.

[9] D. Vande Ginste, D. De Zutter, D. Deschrijver, T. Dhaene, P. Manfredi, and F. Canavero, "Stochastic modeling-based variability analysis of onchip interconnects," IEEE Trans. Compon. Packag. Manuf. Technol., vol. 2, no. 7, pp. 1182-1192, July 2012.

[10] M. Chernobryvko, D. Vande Ginste, and D. De Zutter, "A two-step perturbation technique for nonuniform single and differential lines," IEEE Trans. Microw. Theory Techn., vol. 61, no. 5, pp. 1758-1767, May 2013.

[11] U. R. Patel, S. V. Hum, and P. Triverio, "A magneto-quasi-static surface formulation to calculate the impedance of 3D interconnects with arbitrary cross-section," in 2017 IEEE 21st Workshop on Signal and Power Integrity (SPI), May 2017, pp. 1-4.

[12] M. Huynen, M. Gossye, D. De Zutter, and D. Vande Ginste, "A 3D differential surface admittance operator for lossy dipole antenna analysis," IEEE Antennas Wireless Propag. Lett., vol. 16, pp. 1052 1055, 2017.

[13] Linear Technology. (2016) LT SPice XVII. [Online]. Available: http://www.linear.com/ltpsice/ 